\title{
SODIUM FLUORIDE AS AN ASCARICIDE FOR SWINE RAISED ON THE GROUND
}

\author{
By
}

By Radamee Orlandi ${ }^{1}$ and Fernando E. Armstrong. ${ }^{2}$

Parasitism in livestock is one of the major problems encountered by the animal husbandman in Puerto Rico. Swine are seriously affected with ascarides in this island. This is due to several factors some of which are: tropical temperature, relatively high average rainfall (above 70 inches) and, in general, poor management. In Puerto Rico pigs are raised on the ground with no provisions made to avoid or prevent parasitic infestations; and ascarides constitute a constant menace.

Several drugs have been used at one time or another as anthelmintics for swine. Oil of chenopodium has been used extensively since 1918 when Hall and Foster ${ }^{2}$ first employed it as an ascaricide. According to Mote ${ }^{6}$ its use has been hampered by the difficulty of administration and unreliability in certain cases. Hardwood, Jerstad, and Swanson ${ }^{3}$ in 1938, reported on the efficacy of phenothiazine as an anthelmintic for swine. Their results varied from 0 to 100 per cent against ascarides. Phenothiazine has been stated to be an unreliable ascaricide, especially when fed to pigs harboring few ascarides, as reported by Mohler. ${ }^{5}$ Swanson, et al, ${ }^{8}$ reported that the drug is much less effective against immature than mature ascarides.

In 1945, Enzie, Habermann, and Foster, ${ }^{1}$ described the use of different levels of sodium fluoride for killing ascarides in pigs. They found that when one per cent sodium fluoride, mixed with the feed, was given to twenty-six hogs, a 100 per cent ascaricidal efficiency was obtained. More recently Turk and Hale, ${ }^{9}$ experimenting with large number of pigs, have reported on the ascaricidal properties of sodium fluoride. Their conclusions are based on the number of worms removed and subsequent appearance of the animals submitted to different treatments.

The present experiment was designed to test the value of sodium fluoride as an ascaricide for growing pigs kept on infested grounds and to investigate the possible efficacy of the

\footnotetext{
${ }^{1}$ Assistant Bacteriologist.

2 Associate Veterinarian.
} 
drug to keep animals free from ascarides during their growing period, as well as to observe possible toxicity after repeated treatments.

\section{Materials and Methods}

Two trials were conducted to study the ascaricidal effect of sodium fluoride. The first trial also included the use of phenothiazine.

Trial 1 .- Thirty pigs, ranging in weight from 16 to 34 pounds (averaging 26 lbs.), and about two months of age, were divided into three groups of ten pigs each. The breeding of all groups was similar, litter mates being distributed among the groups in most cases. All of the pigs were kept in a $1 / 2$-acre-infected lot at all times except when groups " $\mathrm{B}$ " and " $\mathrm{C}$ " were separated periodically for a few hours for treatment. Individual weights were taken at the beginning and end of the trial. All pigs were self-fed throughout the trial.

Group "A" served as control. Group "B" was weighed every three weeks and given one meal containing $0.2 \mathrm{gm}$. of phenothiazine per pound of body weight. Group " $C$ " received a treatment of one meal containing sodium fluoride at the rate of 1 pound NaF per 100 pounds concentrate feed, every three weeks.

Trial II.-Was conducted during one year, following Trial I. The conditions were similar except that litter mates were used throughout, sodium fluoride was given at 4-week intervals, and the chances of infection were increased by furnishing a mud wallow.

Egg counts were made per gram of fresh feces secured directly from the rectum of each pig and by using the modified Stoll technique recommended by Hawkins. ${ }^{4}$ Except for a short period at the start of the first trial, differential egg counts were made for ascarid and non-ascarid eggs.

At the end of each trial the hogs were slaughtered and visceral examinations made. In Trial I, some pigs from each of the three groups were spared and reincorporated into the breeding herd. One animal of group " $\mathrm{A}$ " died soon after the experiment was started.

All data were statistically analyzed by methods outlined by Snedecor.? 


\section{EXPERIMENTAL RESULTS}

The results for Trial I are given in tables 1, 2, 3 and 4 .

Although differential counts were not made at the start of the trial, pigs of all groups were shedding ascarid eggs. Total egg counts prior to October 3, 1946 for groups "A", "B" and "C" showed no statistically significant differences. At no time did the numbers of non-ascarid eggs differ statistically among the groups in Trial I. On October 3, 1946 the ascarid egg count for group "C" was statistically significantly lower than for groups "A" and "B". Further statistical treatment for the number of ascarid eggs for Group $\mathrm{C}$ was not made as the numbers were so low. At no time did groups "A" and " $B$ " differ statistically in number of ascarid eggs.

The results of the visceral examination are shown for Trial I in table 4. By the use of a chi square test it was found that groups " $B$ " and " $C$ " contained statistically significantly fewer pigs harboring ascarides than group $A$; and that groups $B$ and $\mathrm{C}$ did not differ significantly. As previously mentioned, some hogs from each group were kept for breeding purposes and not slaughtered. As shown in table 2, two of the three hogs of group B, not slaughtered, were casting sufficiently large numbers of ascarid eggs to imply the presence of ascarides in their intestines. The two pigs of group "C" which were not killed were apparently free of ascarides. Thus, the probable numbers of infected hogs for groups " $B$ " and " $C$ " were four and 0 , respectively. This apparent difference in the number of pigs dropping eggs is extremely important where the object of the treatment is not only to clear the pigs but to prevent continual reinfection of the ground.

Data collected on growth rates for groups "A", "B" and "C" showed that the mean growth rates for the various groups did not differ significantly.

Trial II.-The second trial gave results similar to those found in the first trial (tables 5 and 6 ). 


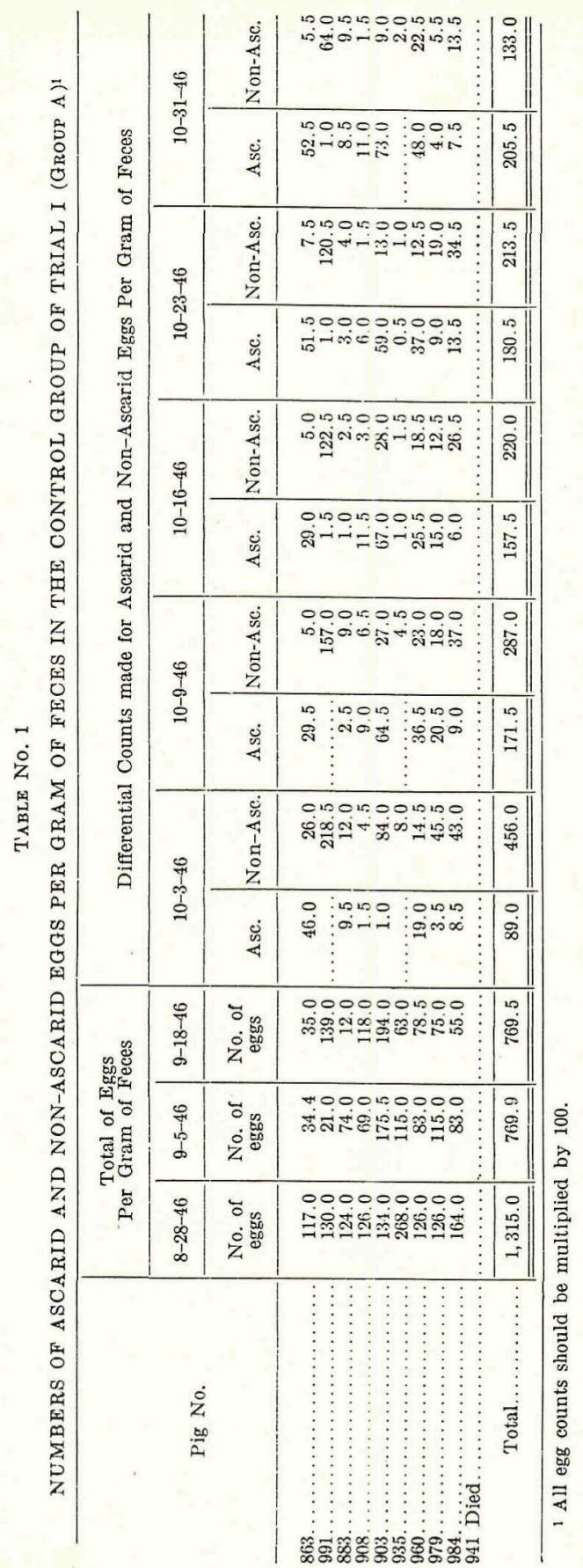




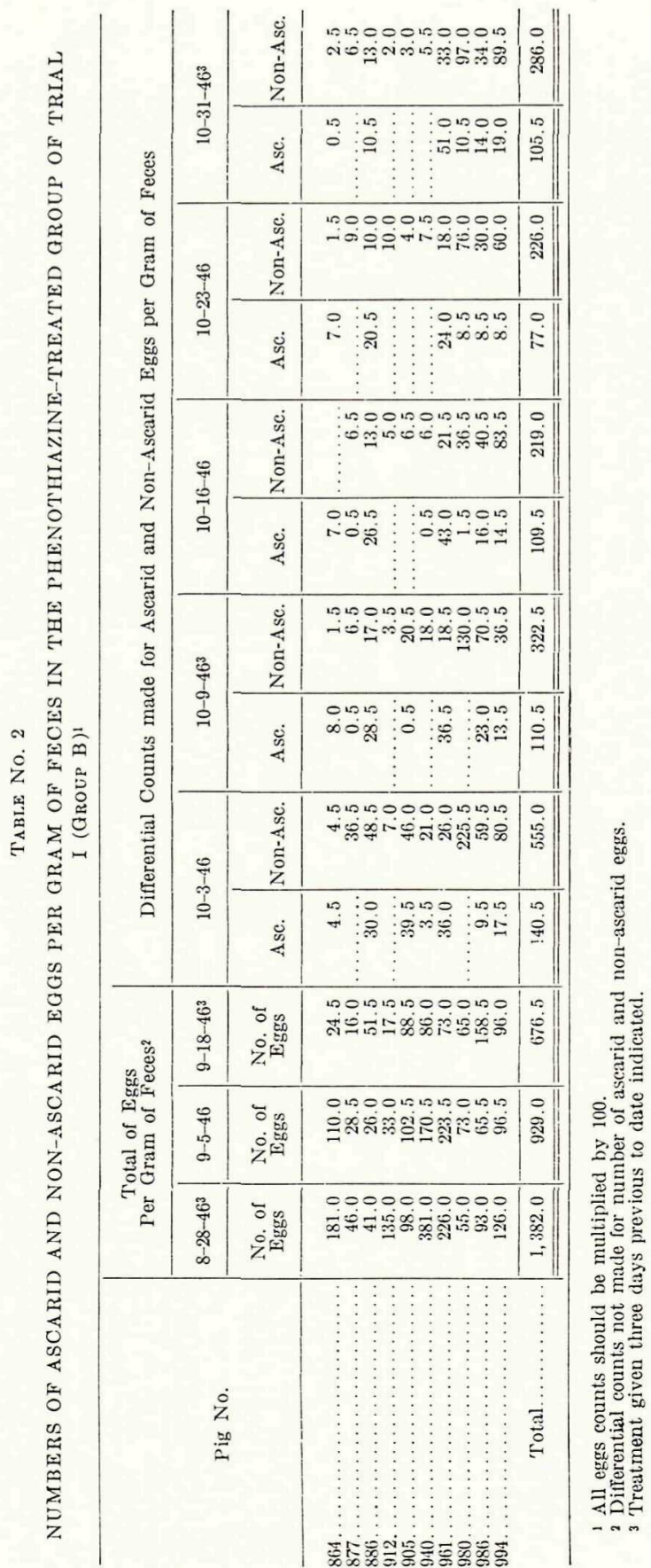




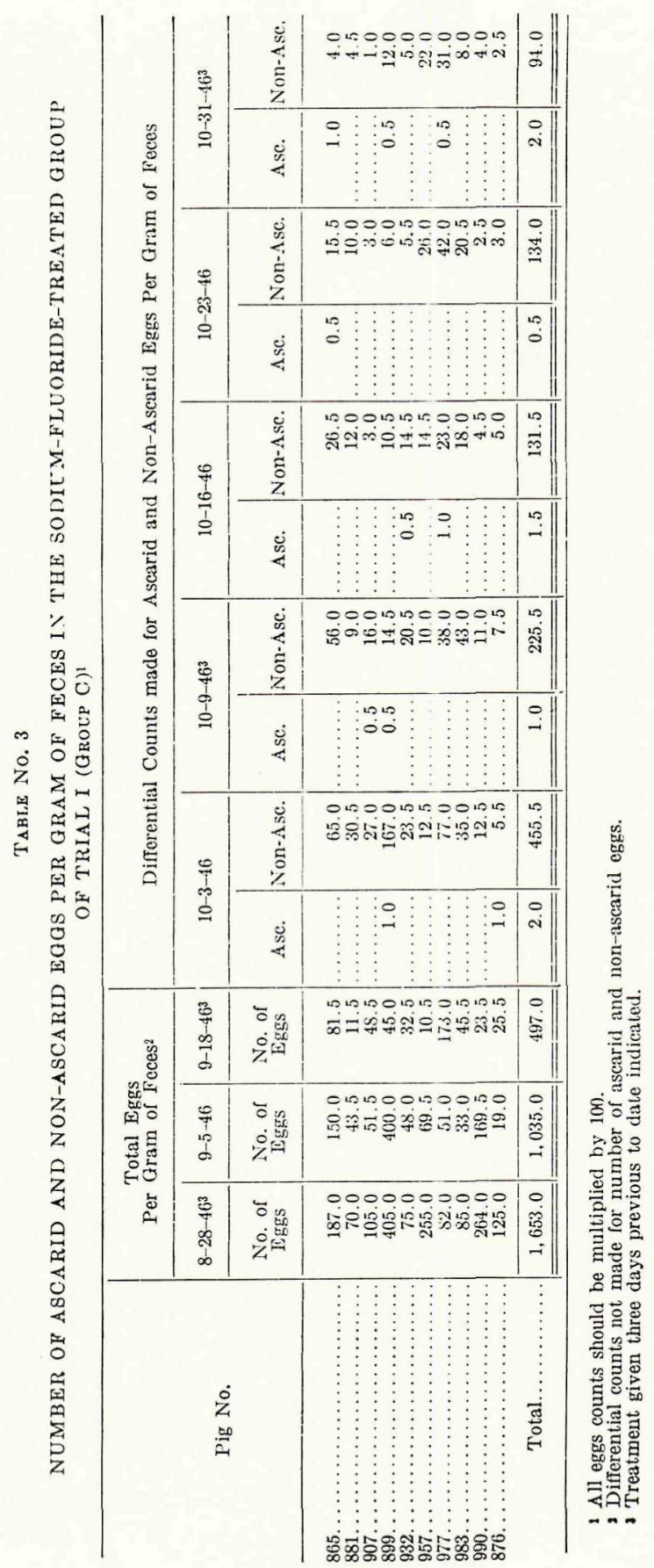




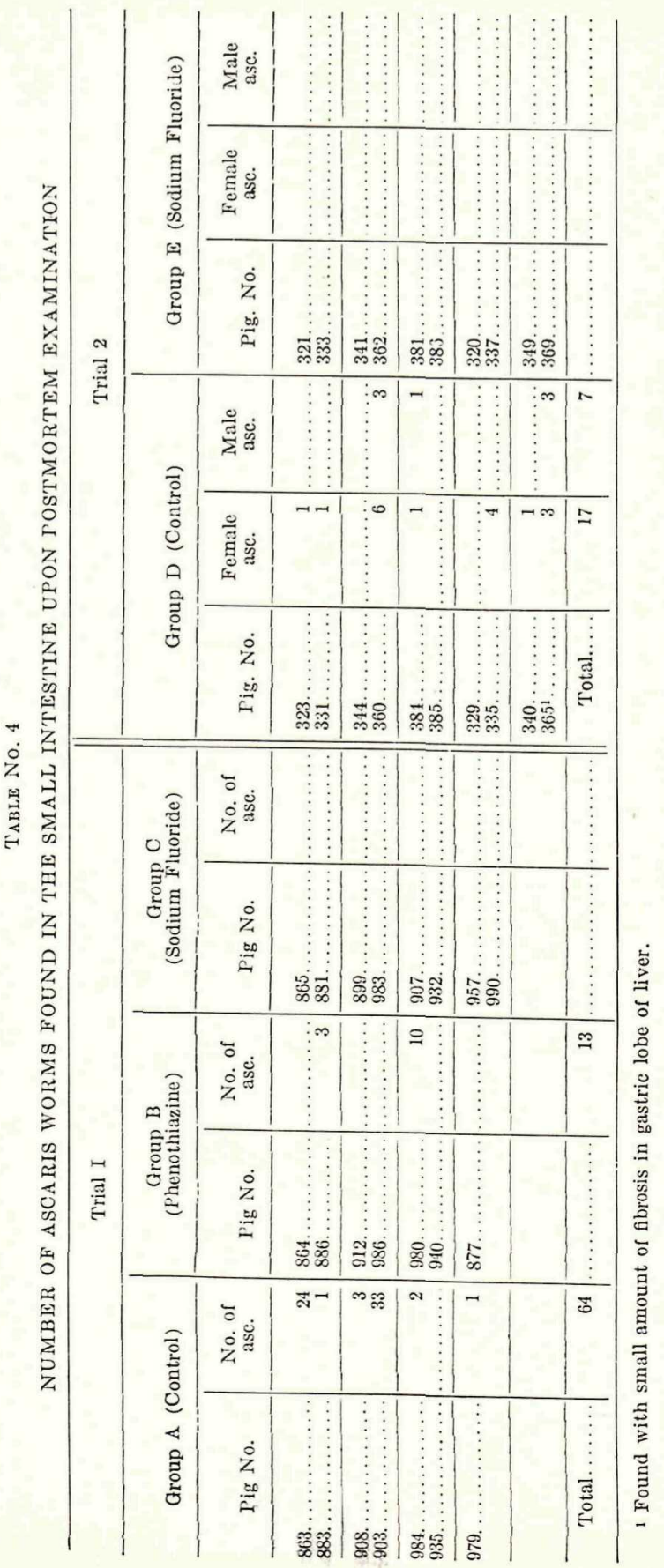




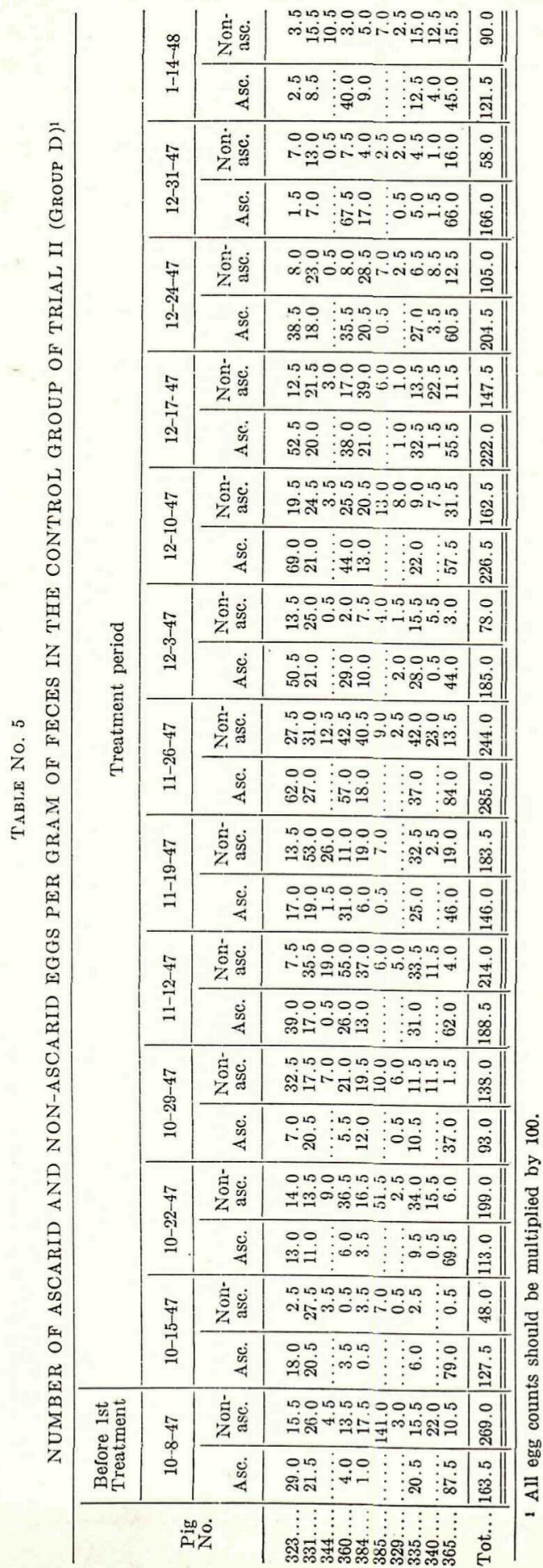




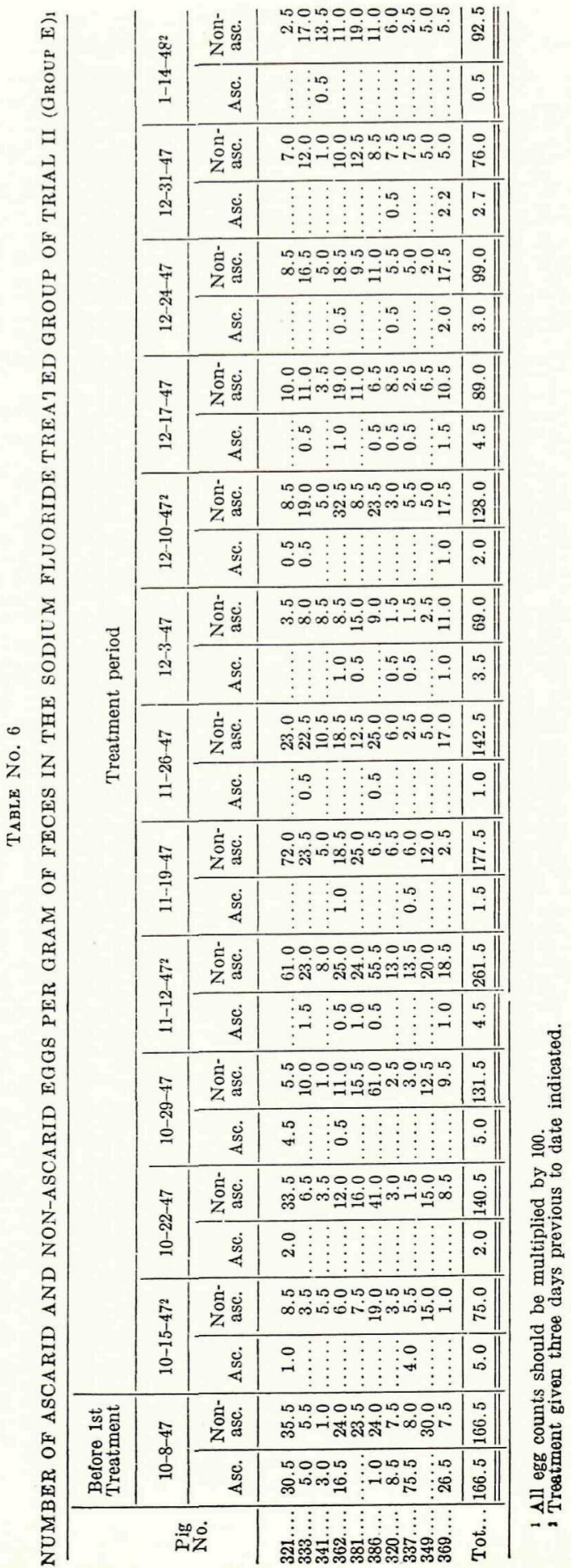


Prior to the first treatment with sodium fluoride, there were no statistically significant difference in either ascarid or nonascarid egg counts between groups " $D$ " and " $E$ ". The data for total ascarid eggs from the time treatments were started to the end of the experiment, exhibited a highly significant difference between the sodium-fluoride-treated and the control pigs (tables 5 and 6). On the other hand, the data for total nonascarid eggs of the sodium-fluoride-treated and control groups collected during the same period (treatment period) showed no statistically significant difference between the two groups.

Upon slaughter, the small intestines of the sodium-fluoridetreated animals (group "E") were found free of ascarid worms, while seven of the ten control pigs (group "D") were infested with a total of 24 ascarides (table 4). All viscerae (groups "E" and "D") were found normal except one pig in group "D" (pig No. 365) which had a small amount of fibrosis in the gastric lobe of the liver. This pig, as will be noticed from table 5, shed the highest number of ascarid eggs throughout the experimental period.

Data were also collected for the growth rate of the two groups studied in this second trial. The growth rates were similar to those obtained in Trial I. The difference in the rate of gain between groups " $D$ " and "E" was statistically non-significant.

\section{DISCUSSION}

The results obtained demonstrate that the pigs given sodium fluoride were apparently free of ascarides for the duration of the trials as ascertained by differential egg counts. Upon slaughter, they were found to be free of ascarides. The ascarid eggs found in the feces of some treated animals were nonembryonated eggs and were probably eggs that were engulfed in the noninfective stage.

Phenothiazine did not exhibit satisfactory ascaricidal properties as shown by the egg counts of table 2 and the recovery of adult worms from the small intestines as shown in table 4. Phenothiazine, in the concentration used, did not prevent infection as shown by pig No. 980 (table 2) which, although free of ascarid eggs on October 3 and October 9, indicated an increasing amount of eggs shed up to the end of the trial and which was found to have ten adult worms when slaughtered. 
In addition, a study of the data of table 2 indicates that the phenothiazine did not consistenly lower the number of ascarid eggs.

The data secured in this study indicate that sodium fluoride is a more satisfactory ascaricide than phenothiazine, at the dosage used. The sodium fluoride proved effective after one treatment. This was shown by the data collected for the number of ascarid eggs shed before and after the first treatment (see ascarid egg counts on October 8 and October 15, 1947 in table 6). No injurious or toxic effects treatment (see ascarid egg counts on October 8 and October 15, 1947 in table 6). No injurious or toxic effects were noted when the use of the oneper-cent mixture with the feed was given every 3 or 4 weeks for periods of 3 to 4 months. Weight gains were not affected by the drug treatments: the control and the sodium-fluoridetreated pigs made similar weight gains. Sustained use of the drug kept the pigs free of adult worms. This is of invaluable advantage where lots are heavily infested and poor management practices prevail. First, there will be fewer adult parasites living at the expense of the feed provided for the growing pigs and secondly, there will be a progressive decrease of chances of infection as a result of having progressively fewer fertile eggs deposited upon the ground.

In relation to the parasitic organisms present, sodium fluoride seemed to be a specific for ascarides. No differential counts were made for non-ascarid eggs but the following types were the most commonly found: Strongyloides, Oesophagostomum, Hyostrongylus, Globocephalus and Trichuris. As the number of these eggs did not decrease as the result of treatment, the sodium fluoride presumably had little or no effect on those organisms.

It may also be stated that in addition to being a reliable ascaricide, sodium fluoride is very economical to use and easy to administer. The mixture of feed and sodium fluoride was found to be palatable.

\section{SUMMARY AND CONCLUSIONS}

Two trials were conducted to test the value of sodium fluoride as an ascaricide for growing pigs kept on infested grounds. The ability of the drug to kep animals free from ascarides and the possible toxicity upon repeated treatment were also studied.

Three groups of animals similar as to breeding, weight and age were used in Trial I. Group "A" served as control. Group 
" $\mathrm{B}$ " received $0.2 \mathrm{gm}$. of phenothiazine per pound or bodily weight and group "C" one per cent sodium fluoride mixed with ground feed. Except for slight variations in the procedure and the elimination of the phenothiazine treatment, Trial II was conducted in the same manner.

The effectiveness of the different treatments given during the experimental period was measured by statistical analyses of the number of ascarid and non-ascarid eggs per gram of fresh rectal feces secured once every week, by the weight gains made by each animal, and by the number of Ascaris worms found upon visceral examination.

The results obtained suggest that sodium fluoride at the rate of one per cent mixed in the feed every three weeks is a very satisfactory drug for killing Ascaris lumbricoides suis found in growing pigs raised on the ground. When fed repeatedly to growing pigs for periods of from 3 to 4 months it was effective in keeping the animals clean, with no toxic effects whatsoever.

Phenothiazine at the rate of $0.2 \mathrm{gm}$. per pound of bodily weight administered every three weeks was found to be unreliable as an ascaricide.

The data obtained during the two trials made suggest that sodium fuoride is specific against Ascaris lumbricoides suis.

\section{ACKNOWLEDGMENTS}

The authors acknowledge the assistance given by Dr. R. E. Comstock and Mr. J. I. Cabrera in the statistical analyses of the data. They are also indebted to Dr. W. W. Green, Head, Animal Husbandry Department, Puerto Rico (University) Agricultural Experiment Station, for his suggestions and assistance in the preparation of the manuscript.

The swine were provided and maintained by the cooperation of Mr. A. González Chapel and Mr. T. H. Quiñones.

\section{REFERENCES}

1. Enzie, F. D., R. T. Haberman, and A. O. Foster. A Comparison of Oil of Chenopodium, Phenothiazine and Sodium Fluoride as Anthelmintics for Swine. Jour. A.V.M.A., 107, (1945):57-66.

2. Hall, IM. C., and W. D. Foster. Efficacy of Some Anthelminties. Jour. Agric. Res., 12, (1918):397-447

3. Harwood, P. D., A. C. Jerstad, and I. E. Swanson. The Efficacy of Phenothiazine for the Removal of Ascarids and Nodular Worms from Swine. Jour. Parasitol., 24, (1938): suppl. 16-17. 
4. Hawkins, P. A. Laboratory Manual (Parasitol. 307 a). Mimeographed by the Mimeograph Dept., Michigan State College, East Lansing, Mich., (1945): appendix pp. iii.

5. Mohler, J. R. Treatment for the Removal of Parasites. Rep. of the Chief of the Bureau of Animal Industry (1940):87-90.

6. Mote, D. C. Anthelmintic Experiments with Hogs. Ohio Agric. Exp. Sta. Bul., 378, (1924):153-182.

7. Snedecor, G. W. Statistical Methods. 4th. ed., The Collegiate Press, Inc., Ames, Iowa, 1946.

8. Swanson, L. E., P. D. Harwood, and J. W. Connelly. Phenothiazine as an Anthelmintic for the Removal of Intestinal Worms from Swine. Jour. Am. Vet. Med. Ass. 96, (1940):33-338.

9. Turk, R. D. and F. Hale. Observations on the Use of Sodium Fluoride as an Ascaricide in Swine. Jour. Am. Vet. Med. Ass., 112, (1948): $363-366$. 